\title{
Overview of a
}

Reconfigurable Simulator for Main Control Room Upgrades in Nuclear Power Plants

\section{Annual Meeting of the Human Factors and Ergonomics Society}

\author{
Ronald L. Boring
}

U.S. Department of Energy

National Laboratory

operated by

Battelle Energy Alliance

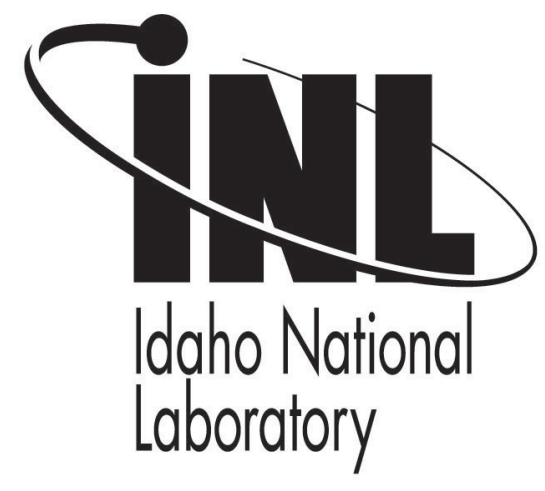

This is a preprint of a paper intended for publication in a journal or proceedings. Since changes may be made before publication, this preprint should not be cited or reproduced without permission of the author. This document was prepared as an account of work sponsored by an agency of the United States Government. Neither the United States Government nor any agency thereof, or any of their employees, makes any warranty, expressed or implied, or assumes any legal liability or responsibility for any third party's use, or the results of such use, of any information, apparatus, product or process disclosed in this report, or represents that its use by such third party would not infringe privately owned rights. The views expressed in this paper are not necessarily those of the United States Government or the sponsoring agency. 


\title{
OVERVIEW OF A RECONFIGURABLE SIMULATOR FOR MAIN CONTROL ROOM UPGRADES IN NUCLEAR POWER PLANTS
}

\author{
Ronald L. Boring \\ Idaho National Laboratory, Idaho Falls, ID 83415, USA
}

\begin{abstract}
This paper provides background on a reconfigurable control room simulator for nuclear power plants. The main control rooms in current nuclear power plants feature analog technology that is growing obsolete. The need to upgrade control rooms serves the practical need of maintainability as well as the opportunity to implement newer digital technologies with added functionality. There currently exists no dedicated research simulator for use in human factors design and evaluation activities for nuclear power plant modernization in the U.S. The new research simulator discussed in this paper provides a test bed in which operator performance on new control room concepts can be benchmarked against existing control rooms and in which new technologies can be validated for safety and usability prior to deployment.
\end{abstract}

\section{BACKGROUND ON REACTOR LIFE EXTENSION}

The Light Water Reactor Sustainability (LWRS) Program is a research, development, and deployment program sponsored by the United States Department of Energy (U.S. DOE). The program is operated in close collaboration with industry research and development $(\mathrm{R} \& \mathrm{D})$ programs to provide the technical foundations for licensing and managing the long-term, safe, and economical operation of nuclear power plants (NPPs) that are currently in operation. Nuclear power accounts for $20-25 \%$ of current baseload electricity generation in the U.S.; yet, replacement technologies including renewable energy or new plants have been slow to materialize. Without suitable replacements in place or in planning, it is imperative to ensure the continued safe supply of electricity through the current fleet of power reactors. The LWRS program focuses on research that contributes to the national policy objectives of energy and environmental security.

One of five principal LWRS R\&D pathways addressing the Strategic Program Goals is Advanced Instrumentation, Control, and Information Systems Technologies (Hallbert et al., 2009). This pathway uses a scientific knowledge basis and advanced phenomenological modeling to establish advanced condition monitoring and prognostics technologies for use in understanding the aging of systems, structures, and components of NPPs. Information system technology enhancements will be developed to demonstrate knowledge migration and regulatory compliance.

Advanced instrumentation and control (I\&C) technologies are needed to support the safe and reliable production of power from nuclear energy systems during sustained periods of operation up to and beyond their expected licensed lifetime. This requires the development and eventual implementation of new process control capabilities in existing nuclear assets. It also requires that approaches be developed and proven to achieve sustainability of I\&C systems throughout the period of extended operation. The strategic objective of the DOE's Advanced Instrumentation, Information, and Control Systems Technologies research and development $(R \& D)$ pathway is to establish a technical basis for new technologies needed to achieve safety and reliability of operating nuclear assets and to implement new technologies in nuclear energy systems. This will be achieved by carrying out a program of R\&D to develop scientific knowledge. One of these areas of R\&D is new methods for visualization, integration, and information use to enhance state awareness and leverage expertise to achieve safer, more readily available electricity generation, which includes new or enhanced control systems.

Idaho National Laboratory (INL) is working closely with nuclear utilities to develop technologies and solutions to help ensure the safe life extension of current NPPs. One of the main areas of focus is control room modernization. The current analog control rooms are nearing the end of their usable life, and it is difficult for utilities to maintain them. Using its reconfigurable control room simulator, INL serves as a neutral test bed for implementing new control room system technologies. The INL simulator test bed, which can be used to develop and test the implementation of new digital control room systems, serves as a resource for testing emerging technologies for their application in NPP control rooms.

\section{NUCLEAR POWER PLANT SIMULATORS}

\section{General Introduction to Simulators in Nuclear Energy}

Typically, simulators serve to train operators on the proper use of workplace devices, but simulators are also frequently employed in research to evaluate human performance. Simulator technology for domains such as aviation emerged in the 1930s with the invention of the Link Trainer, a mockup plane that allowed pilots in training to learn to manipulate flight controls in a rudimentary manner (American Society of Mechanical Engineers, 2000). It was not until considerably later-with advances in computing technology - that mathematical system models and computer generated imagery could be harnessed to create realistic, virtual flight simulations.

A similar course was followed for NPPs-initial, nonoperational hardware mockups of control room panels 
used by the U.S. nuclear Navy and plant vendors gave way to entire control room simulators with functional control panels that connected with underlying thermal-hydraulic code. NPP simulators evolved from static training representations to interactive, operational systems that could be used to train and test reactor operators' knowledge of plant states and scenarios. An NPP simulator today consists of a computing system to mimic the function of the plant and a physical representation of the control room that allows operators to monitor simulated plant states and control plant functions.

Historically, by 1973, fully functional simulators had been developed that had all the controls, dials, gauges, lights, switches, and recorders found at the host plants. The early simulators attempted a high degree of physical realism by providing a reasonably faithful replica of the control rooms found at actual plants. Frequent updates to plant control room hardware, due to the changes in technology used at operating plants, meant that the simulator had to be updated and reprogrammed on a frequent basis, making the simulator's replication of the plant control room difficult to maintain. Functionally, the underlying computing system had limited success at achieving realistic scenario progressions, because only a limited number of plant scenarios could be accommodated by the underlying computing hardware. Nonetheless, these early simulators served a vital role in training and licensing crews.

A 2004 report by the International Atomic Energy Agency (IAEA) highlights the historic development of training simulators. Beginning in the 1970s, computerized control room simulators were put in place at centralized facilities to help train control room operators. These simulators were limited by a lack of fidelity in terms of control panel layouts and underlying thermal-hydraulic code, making them useful for teaching basic plant principles but less useful for plant-specific training. By the 1980s, the fidelity and availability of simulators had greatly increased, and by the 1990s, it became commonplace internationally for each plant to have a high-fidelity plant-specific training simulator. In the US, a requirement for training simulators at every plant was introduced so that the U.S. Nuclear Regulator Commission (2001) could license operators with a high degree of confidence, which also enabled reactor operators to train on unusual or unlikely events.

The IAEA (2003) defines four different types of plant simulators. These include:

- Basic principles simulator-provides a simulation of general concepts relevant to the operation of a plant without providing a faithful mockup of a specific plant

- Full-scope simulator-is a faithful replica of a specific plant control room and its operations

- Other-than-full-scope control room simulator-closely mimics a plant but deviates from its human-machine interface

- $\quad$ Part-task simulator-only models specific systems and interfaces of a plant.
In practice, the term training simulator is synonymous with a full-scope simulator as would be found at an NPP. All simulator types may be used as part of an effective training regime, but there has been increased emphasis on and requirements for training in full-scope simulators.

Training simulators at NPPs are in high demand. Despite high plant use of training simulators, there remains an ongoing and equally important need to use simulators for understanding operator performance. The need for research on control room crews serves to maintain and enhance the safety at current plants and to document operator interaction with emerging control room technologies. Yet, the availability of training simulators is severely limited, and there remains the inability to reconfigure their use for research purposes. As such, control room simulators have been created to serve the primary purpose of conducting research independent of training. These are research simulators.

\section{The Need for Dedicated Research Simulators}

Research can be performed using both training and dedicated research simulators. The differences are centered on the types of studies that can be conducted and the types of data that that can be collected from the studies.

Where the aim is to collect human performance information from actual crews in current control room configurations, the training simulator offers a logical first stop. Participation in simulator research studies affords a unique opportunity to investigate factors affecting crew performance in current control rooms. Practically speaking, over time, such studies may be used to establish new industry best practices and to improve crew preparedness for unusual plant events. From a research perspective, findings from training simulator studies may inform new or improved methods of human performance or human reliability analysis, or be used to develop a more realistic representation of normal crew performance. Such research may also drive recommendations for the implementation of next-generation control room interfaces, based on principles of crew performance in current control rooms.

However, the practical limitations of training simulators for research must be understood:

- $\quad$ Limited availability. Training simulators have as their first priority the training of crews. Research studies may be scheduled as available, but they must not interfere with required training exercises. For this reason, only research studies that align closely with training tasks are well suited for training simulators. Crews, trainers, and the simulator facility are limited commodities at the plant, and research studies should complement their primary purpose.

- Simulator inflexibility. The flexibility to manipulate plant parameters and operational situations is limited in the training simulator. For particular research questions related to crew performance, it may be desirable to configure the plant parameters in an unusual way (e.g., 
multiple simultaneous faults). While this level of control should be available in training simulators, the ease with which such manipulations can be made may be limited by the need to create readily configurable scenarios appropriate to training.

- Limited data collection. The ability to collect different types of data in the naturalistic setting is restricted. Primarily observational and plant log data may be collected, and advanced data collection techniques such as noted in Tran et al. (2007) are not easily or unobtrusively retrofitted to the training simulator.

- Fixed Human-System Interface (HSI). Training simulators are purpose built to mimic the actual HSI of a specific plant. As such, training simulators are not typically well suited for exploratory studies of novel control room interface elements. Though training simulators may be suitable for implementation of equipment upgrades at the plant (e.g., phasing in new control panels and training crews on them prior to installation in the actual plant control room), they are not generally suited for trying out new configurations.

The above limitations of training simulators for research illustrate the importance of maintaining and championing dedicated research facilities like the INL's Human System Simulation Laboratory, a simulator for control room simulation. Dedicated research simulators are ideal for:

- $\quad$ Scheduling flexibility. Research simulators are generally not in as heavy rotation for use as plant training simulators. Depending, of course, on the number of studies being conducted, it is possible to schedule research simulators for longer periods of time and with greater scheduling flexibility.

- Configuration flexibility. Research simulators offer maximum control over plant parameters and do not have to be limited to a specific plant. In fact, research simulators may be reconfigured to different types of plants, including advanced plants that are still under development. For example, a research simulator may be easily reconfigured to be either a pressurized water reactor or a boiling water reactor. Further, a research simulator may be configured to be functionally equivalent to specific plants within those plant types. It is also possible to couple a research simulator to hard panels that faithfully mimic analog control rooms. A research simulator may also be reconfigured in task- or function-specific I\&C, such as evaluating operator performance in response to digital alarm systems.

- Data flexibility. Research simulators may allow the collection of observational data similar to those data collected in training simulators. In addition, it is possible to collect data such as physiological measures and eye tracking that require specialized equipment not easily retrofitted to training simulators.

- $\quad$ Crew flexibility. Reconfigurability makes it possible to study crews from different plants within the same study. The simulator may be configured to match the home plant very closely, or a hybrid approach may be adopted, whereby crews operate on a generic plant that is similar to but not identical to their home plant. For example, studies involving different crews are important for understanding operational culture (Heimdal, 2007).

Of course, limitations to using research simulators exist, not the least of which is the feasibility of securing qualified reactor operators to participate in studies. Beyond that, the primary limitation is the generalizability of the results:

- Generalizability of the control room. In a research simulator, the HSI may not be a direct replica of a specific physical plant but rather a functional equivalent. Evidence suggests that simulators that are functionally similar will generate comparable results to each other (Stanton, 1996). However, some features of research simulators can introduce subtle differences between the simulator and the actual plant. Such differences are endemic to research simulators, although measures are being undertaken at the INL to ensure maximum compatibility of the research simulator to actual plant control rooms.

- Generalizability of the crews. In part, differences in operational culture may make it difficult to generalize simulator study results across crews. There are differences in crews due to the nuances of plant design, differences in training, and other factors. It can be difficult to generalize the findings from one plant or crew to another. For this reason, INL is focusing on general principles and findings rather than plant-specific findings for its longer-term research. Of course, beyond general principles, research conducted in support of a specific plant will make maximum use of the crews connected to that plant.

\section{HUMAN SYSTEM SIMULATION LABORATORY}

At the heart of a full-scope plant simulator is a series of system models that interact to create a realistic model of plant behavior, including thermal-hydraulic software modeling using RELAP, a vendor-specific simulator platform (e.g., simulator software development packages by GSE, WSC, and L-3), and a plant-specific model executed on the simulator platform. These models combine to form the back end called the engineering simulator. The engineering simulator interfaces with the front-end simulator, which consists of the control room HSI that the operator uses to understand plant states and control plant functions. The front-end simulator may take many forms such as an analog hard panel system found in typical training simulators or a digital soft control system found in some foreign plants and research simulators. Digital soft control systems (also sometimes referred to as glass top panels) may take the form of mimics to analog plant I\&C or may represent advanced I\&C that incorporates features such as overview displays and information rich trending displays.

The INL Human System Simulation Laboratory is a platform-neutral environment intended for full-scope and part-task testing of operator performance in various control 
room configurations. Currently, plant-specific simulators are coupled to the existing configuration of the plant and are impractical or difficult to reconfigure to test new designs. The INL facility is not limited to a particular plant or even simulator architecture. It currently supports engineering simulator platforms from multiple vendors using digital interfaces. With reconfigurability, it is possible to switch the I\&C - not just to digital panels but also to different control modalities such as those using greater plant automation or intelligent alarm filtering. Current efforts are centered around building out a Combustion Engineering full-scope simulator that is an exact digital replica of the plant control room at an operating NPP. The intent is that licensed plant operators will be able to use the facility as a research simulator, because there is limited availability of the plant training simulator.

The international nuclear community has for over 25 years been working with Halden Reactor Project in Norway to run control room simulator studies. The Halden Reactor Project has a reconfigurable control room simulator called the Halden Man-Machine Laboratory (HAMMLAB) that can model advanced control rooms for boiling water and pressurized water reactor configurations. These studies, which avail themselves of licensed plant operating crews from Swedish or Finnish plants, are used to determine crew behavior in a variety of normal and off-normal plant operations. The findings are ultimately used to guide safety considerations at plants and to inform human factors for both regulators and industry.

Recently, there has been a strong desire to have access to similar facilities in the U.S. The HAMMLAB facilities are strictly research facilities. They are not used for training, because they do not map to any current plant. Much of the technology used at HAMMLAB is cutting-edge and is not part of standard plant control rooms. For example, the HAMMLAB control room is all digital, featuring large overview displays, menu-based soft controls, and scrolling alarm lists instead of annunciator displays (see Figure 1). The HAMMLAB simulator is optimized for testing and improving new control room technologies, but it is not configured to mimic current control rooms. INL's facilities serve to complement HAMMLAB by providing a simulator focused on modernization of existing NPPs.

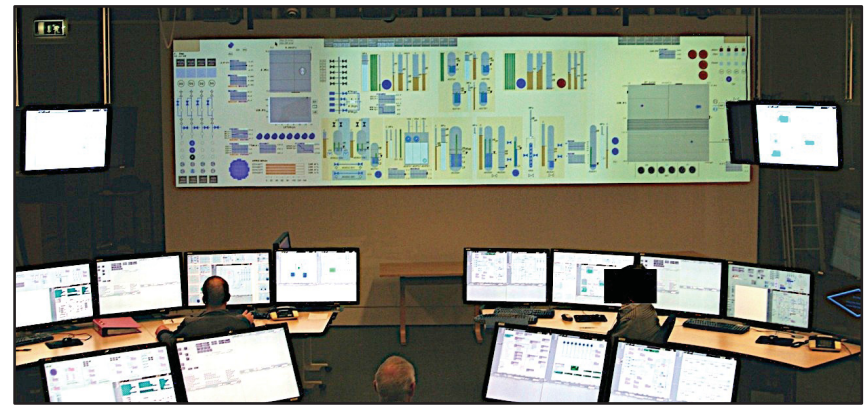

Figure 1. Advanced HAMMLAB control room.
Of the 104 currently operating nuclear power plants in the U.S., not one has successfully completed a full-scale conversion from analog to digital I\&C in the main control room. The control rooms at current NPPs feature analog I\&C technology in many cases dating to the 1970s (see Figure 2). Although this aging control room technology is adequately maintained to ensure reliability and safety, the cost to maintain such obsolete equipment is approaching or even exceeding the cost of replacement. Yet, there exist significant financial and regulatory hurdles to modernize control rooms, which has slowed vendors to provide comprehensive solutions that meet industry needs. The time required to perform a full-scale control room upgrade is significant, and the cost of loss of production for utilities reaches up to $\$ 2$ million per day for a commercial reactor. Thus, wholesale modernization in the form of complete replacement of these control rooms is not likely in the U.S., and plants are adopting a piecemeal or system-by-system approach to upgrades.

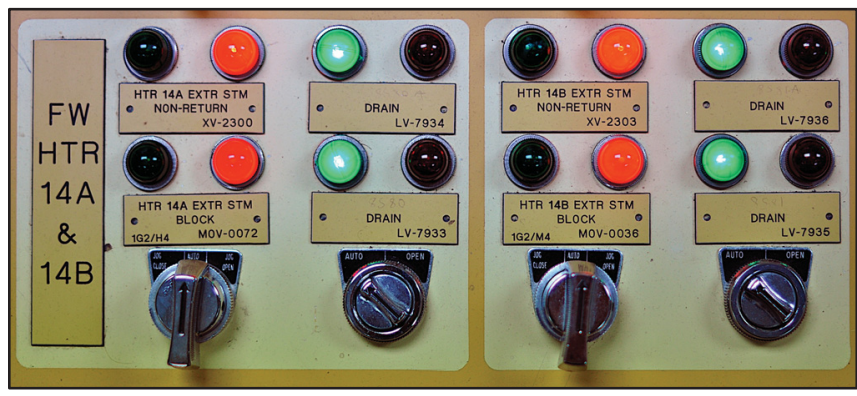

Figure 2. Example panel from current control room.

There exists no research simulator configured specifically to address the redesign of legacy control rooms in the U.S. nuclear industry. The INL Human System Simulation Laboratory is designed to mimic existing control rooms, complete with analog gauges and manual switch controls. The configuration employed uses a so-called glass top simulator comprised of three 46-inch LCD displays (see Figure 3). The lower two displays feature touch screens to allow operators interaction with virtualized controls. The upper display, which is out of operator reach, is an LCD screen without touch interaction. In a faithful mimic of a conventional control panel, the lower and upper panels are mounted at slight angles, with the lower display configured as a bench top area.

At the INL Human System Simulation Laboratory, the glass top panels are linked together in a horseshoe shape that approximates the shape found in current control rooms. A total of 15 glass top panels are linked together, representing faithful high resolution mimics of hardware panels found in current control rooms. Each glass top panel serves as a client to a central simulator model. The panels represent functions related to plant auxiliary services, electrical energy, waste heat removal, primary and secondary energy, reactor support systems, and safety systems. The design requires that all analog I\&C be represented on the control 
panels and that multiple operators may work on the composite simulator at the same time.

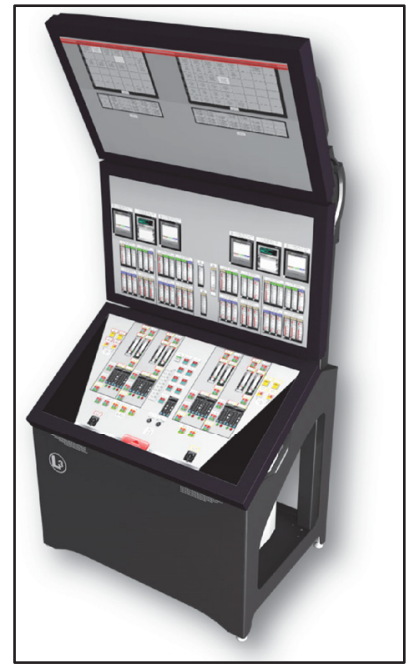

Figure 3. Glass top panel used for simulator.

The key advantage of mimicking current control rooms comes from the ability to implement prototypes of new digital function displays into the existing analog control environment. Prior to full-scale deployment of technologies such as control room upgrades, it is essential to test the performance of the system and the human operators' use of the system in a realistic setting. In control room research simulators, upgraded systems can be integrated into a realistic representation of the actual system and validated against defined performance criteria. In this manner, control room upgrades are being designed, usability tested, and safety validated without the need to use the plant's training simulator.

\section{CONCLUSIONS}

Instead of buying commercial-off-the-shelf digital replacement systems or contracting custom systems that are developed offsite and only later integrated into the control room, the present approach uses the INL replica simulator as the development platform and test bed. The approach affords considerable advantages over traditional upgrades: (1) the design process is formative, meaning it is possible to change ineffective elements of system design prior to full scale integration; (2) the design process is iterative, meaning it is possible to collect operator feedback at early stages of development and apply insights on operator performance into early-stage redesign; (3) the design process is environmentally driven, meaning it captures and mitigates constraints of the control room and aspects of the conduct of operations that might otherwise hinder successful implementation of an interface; (4) the design process converges on a standard, meaning the development of system-by-system upgrades affords the opportunity to create a style guide that may be used to drive a consistent design across the control room; and (5) the design process is cost effective, meaning it is possible to take advantage of inhouse engineering and human factors expertise to design and evaluate systems as they will actually be used. In this paper, we have discussed the approach of using a reconfigurable, full-scope research simulator for control room modernization projects. Integrated system design with a research simulator proves a relevant approach that goes beyond late stage validation to ensure plant upgrades are usable, safe, and cost effective.

\section{DISCLAIMER}

Portions of this paper were previously published and are included in order to provide suitable background. This work of authorship was prepared as an account of work sponsored by an agency of the United States Government. Neither the United States Government, nor any agency thereof, nor any of their employees makes any warranty, express or implied, or assumes any legal liability or responsibility for the accuracy, completeness, or usefulness of any information, apparatus, product, or process disclosed, or represents that its use would not infringe privately-owned rights. Idaho National Laboratory is a multi-program laboratory operated by Battelle Energy Alliance LLC, for the United States Department of Energy under Contract DE-AC0705 ID 14517.

\section{REFERENCES}

American Society of Mechanical Engineers. (2000). The Link Flight Trainer: A Historic Mechanical Engineering Landmark. New York: ASME.

International Atomic Energy Agency. (2003). Means of Evaluating and Improving the Effectiveness of Training of Nuclear Power Plant Personnel, IAEA-TECDOC-1358. Vienna: IAEA.

Hallbert, B., Persensky, J., Smidts, C., Aldemir, T., and Naser, J. (2009). Report from the Light Water Reactor Sustainability Workshop on Advanced Instrumentation, Information, and Control Systems and Human-System Interface Technologies, INL/EXT-09-16631. Idaho Falls: INL.

Heimdal, J. (2007). Operational Culture Literature Review: Extended Summary, HWR-901. Halden, Norway: OECD Halden Reactor Project.

International Atomic Energy Agency. (2004). Use of Control Room Simulators for Training of Nuclear Power Plant Personnel, IAEA-TECDOC-1411. Vienna: IAEA.

U.S. Nuclear Regulatory Commission. (2001). Nuclear Power Plant Simulation Facilities for Use in Operator Training and License Examinations, Rev. 3, Regulatory Guide 1.149. Washington, DC: U.S. NRC.

Tran, T.Q., Boring, R.L., Dudenhoeffer, D.D., Hallbert, B.P., Keller, M.D., and Anderson, T.M. (2007). Advantages and Disadvantages of Physiological Assessment for Next Generation Control Room Design. Proceedings of the Joint 8th IEEE Conference on Human Factors and Power Plants, Monterey, CA.

Stanton, N. (1996). Simulators: A Review of Research and Practice. In N. Stanton (ed.), Human Factors in Nuclear Safety. London: Taylor and Francis. 